Original Article

\title{
Effects of exercise intervention on arterial stiffness in middle-aged and older females: evaluation by measuring brachial-ankle pulse wave velocity and cardio-ankle vascular index
}

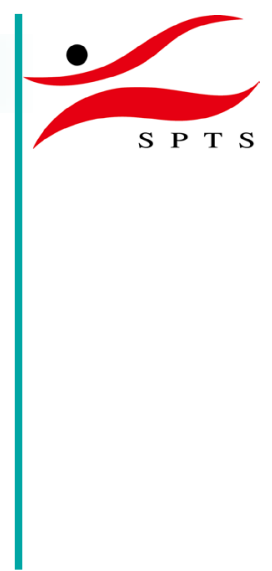

\author{
Hiroya Koshiba, $\mathrm{PhD}^{1)^{*}}$, Etsuko Maeshima, $\mathrm{MD}, \mathrm{PhD}^{2)}$ \\ 1) Faculty of Health and Well-being, Kansai University: 3-3-35 Yamate-cho, Suita-shi, \\ Osaka 564-8680, Japan \\ 2) Graduate School of Health and Sport Sciences, Osaka University of Health and Sport Sciences, \\ Japan
}

\begin{abstract}
Purpose] Brachial-ankle pulse wave velocity (baPWV) and cardio-ankle vascular index (CAVI) are simple, accurate, and reproducible indices of arterial stiffness. However, only a few studies have evaluated the influence of exercise-training baPWV and CAVI on arterial stiffness. Thus, this study aimed to examine the influence of aerobic-training baPWV and CAVI on arterial stiffness. [Participants and Methods] The participants were 20 middle-aged females (age, $60.1 \pm 1.6$ years) who were sedentary non-smokers. The participants underwent an exercise intervention, including a 50-min session of moderate-intensity exercises two or three times a week for 12 weeks. Blood pressure (BP), baPWV, and CAVI were measured before and after exercise. Blood nitrite/nitrate (NOx) was also measured as an index of vascular endothelial function. [Results] BaPWV significantly decreased after exercise, whereas CAVI and blood NOx did not change significantly after exercise. A significant correlation was observed between the decrease in baPWV and systolic BP after exercise. In addition, the participants with higher systolic BP before exercise showed a greater decrease in baPWV after exercise. [Conclusion] These results suggest that BP has a significant role in decreasing arterial stiffness after exercise. Further studies are required to elucidate the similarities and differences between baPWV and CAVI to use them more effectively as assessment parameters for arterial stiffness.

Key words: Exercise, Arterial stiffness, Blood pressure
\end{abstract}

(This article was submitted Aug. 15, 2018, and was accepted Oct. 24, 2018)

\section{INTRODUCTION}

Arteriosclerotic diseases, including cardiac and cerebrovascular diseases, are among the leading causes of death among the Japanese. Arterial stiffness is an important risk factor for arteriosclerotic diseases ${ }^{1)}$ and is an indicator of functional changes associated with atherosclerosis. Evaluation of arterial stiffness helps detect structural changes of atherosclerosis earlier than those detected by intima media thickness or computed tomography. Carotid-femoral pulse wave velocity (cfPWV) had been used as the gold standard for evaluating arterial stiffness for a long time. However, cfPWV was applied to clinical practice in a limited number of cases because it requires an experienced examiner and inguinal exposure. To address these issues, simple, highly accurate, and reproducible measures of arterial stiffness, that is, brachial-ankle pulse wave velocity (baPWV) and cardio-ankle vascular index (CAVI), were developed in the 2000s and have since been widely used as laboratory parameters.

An issue raised with respect to baPWV use includes the difference in the measuring range between baPWV and cfPWV.

*Corresponding author. Hiroya Koshiba (E-mail: koshiba@kansai-u.ac.jp)

(C2019 The Society of Physical Therapy Science. Published by IPEC Inc.

(c) (1) $\odot$ This is an open-access article distributed under the terms of the Creative Commons Attribution Non-Commercial No Derivatives

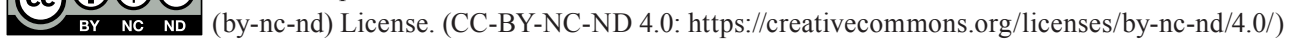


BaPWV measurement is performed not only in the aorta but also in the muscular artery because of the use of pulse waves traveling from the upper extremities to the lower extremities. However, a study has demonstrated a correlation between these two measures $(\mathrm{r}=0.76)^{2}$, and another study demonstrated that baPWV is an excellent predictor of all-cause mortality and cardiovascular accidents ${ }^{3)}$. These observations led to the establishment of baPWV as a measure of functional changes in atherosclerosis.

On the other hand, CAVI was developed following baPWV as a measure of arterial stiffness independent of blood pressure, to compensate for the disadvantages of principle-based measurements of cfPWV and baPWV, which are greatly affected by blood pressure. CAVI is used to quantify arterial stiffness based on the stiffness parameter $\beta$ theory ${ }^{4)}$, with application of the Bramwell-Hill equation. An issue raised with respect to CAVI use includes less evidence indicating the usefulness of this measure for evaluating arterial stiffness, although reported in a few studies ${ }^{5,6}$, as compared to evidence on the usefulness of cfPWV and baPWV.

Previously, many studies on measures of arterial stiffness and exercise, aimed at preventing an increase in arterial stiffness, have been reported. However, there are only a few studies that have compared the effects of exercise training arterial stiffness, as evaluated by simultaneously measured baPWV and CAVI ${ }^{7-11)}$. Li et al. showed a difference in response between baPWV and CAVI after resistance training ${ }^{7)}$. This result indicates the possibility that baPWV is adapted to the changes in blood pressure. Therefore, we had a hypothesis that baPW and CAVI which are indicators of arterial stiffness show the different responses to exercise.This study evaluated the effects of moderate-intensity aerobic exercise training on two different arterial stiffness indices.

\section{PARTICIPANTS AND METHODS}

Participants included 20 middle-aged and older women without exercise or smoking habits who had not previously received oral contraceptives or hormone replacement therapy (60.1 \pm 1.6 years old). Of the 20 participants, 11 were deemed to have prehypertension or hypertension. The participants underwent measurements at two time points: before (pre) and after 12 weeks of aerobic exercise (post). Before the study, all participants received and fully understood written and oral explanations of the objectives, methods, and safety of this study, and then gave written informed consent for study participation. This study was approved as "a study involving human subjects" by the research ethics review committee of Osaka University of Health and Sport Sciences (approval no. 14-35).

Among the body composition measures, height, body weight, body mass index (BMI), and body fat percentage were measured using Body Composition Analyzer InBody 3.2 (BioSpace Inc., Tokyo, Japan).

The measures of arterial stiffness, including baPWV and CAVI, were determined using the Form PWV/ABI device (Omron Colin Co. Ltd., Japan) and Vasera VS-3000 (Fukuda Denshi, Tokyo, Japan), respectively. These devices simultaneously measured systolic blood pressure (SBP) and diastolic blood pressure (DBP). These measurements were performed at least 3 hours after breakfast consumption (Influence of detraining on temporal changes in arterial stiffness in endurance athletes: a prospective study) ${ }^{12}$. Participants were prohibited from strenuous exercise for at least 12 hours before measurement as well as from caffeine consumption after waking up. Before the initiation of measurements, the participants rested quietly in the supine position for 15 minutes at a room temperature (around $24^{\circ} \mathrm{C}$ ). Furthermore, blood concentrations of nitrite/nitrate (NOx) were measured to evaluate the mechanism through which exercise reduces arterial stiffness. The participants were instructed to consume nothing other than water from 21:00 hours on the day before measurement until the measurements were obtained, and their blood samples were collected after at least 12 hours of fasting. Analysis of these samples using high performance liquid chromatography ${ }^{13}$ ) was outsourced to Japan Clinical Laboratories, Inc.

Participants performed 50 minutes of aerobic exercise on a bicycle ergometer (AEROBIKE 75XL II and AEROBIKE $75 X \mathrm{X}$ III, Combi Co. Ltd., Japan) and walking at 50\% of the heart rate reserve, 2-3 times per week (under supervision of an instructor twice per week) for 12 weeks. In addition, physical work capacity at $75 \%$ of heart rate maximum (PWC $75 \%$ HR $\max$ ) was measured pre- and post-intervention to assess general endurance ${ }^{14)}$.

All results of this study were expressed as mean \pm standard deviation. The mean values for each parameter were compared using the t-test. The relationship between the parameters was evaluated using Pearson product-moment correlation coefficient. Multiple regression analysis (stepwise method) was performed using the change in baPWV as a dependent variable to identify the factors contributing to its change. Statistical calculation was performed using IBP SPSS Statistics 23 (IBM SPSS Japan, Inc., Tokyo, Japan), and the statistical significance level was set at $\mathrm{p}<0.05$.

\section{RESULTS}

The characteristics of the participants at two time points (pre and post) are shown in Table 1. SBP and DBP at the post time point were significantly reduced as compared to the respective pre values ( $<<0.01$ and $p<0.05$, respectively). Furthermore, PWC $75 \%$ HR max as a measure of general endurance at the post time point was significantly increased from its pre value $(\mathrm{p}<0.05)$. However, no significant difference was observed in height, body weight, BMI, body fat percentage, or NOx between the pre and post time points. In all participants studied, baPWV at the post time point significantly decreased as compared to its pre value (Pre: $1,420.2 \pm 48.6 \mathrm{~cm} / \mathrm{sec}$ vs. Post: $1,310.0 \pm 39.3 \mathrm{~cm} / \mathrm{sec}, \mathrm{p}<0.001$ ) (Fig. 1); in contrast, CAVI was not significantly different between the pre and post time points (Pre: $7.61 \pm 0.17$ vs. Post: $7.39 \pm 0.29)$ (Fig. 2). The postexercise decrease in baPWV was significantly higher in participants with optimal blood pressure $(50.2 \pm 17.5 \mathrm{~cm} / \mathrm{sec})$ than 
Table 1. Characteristics of participants and changes after the 12 week aerobic exercise intervention

\begin{tabular}{lrc}
\hline & Pre & Post \\
\hline Height $(\mathrm{cm})$ & $155.7 \pm 1.1$ & - \\
Body weight $(\mathrm{kg})$ & $56.8 \pm 1.9$ & $56.3 \pm 1.9$ \\
BMI $\left(\mathrm{kg} / \mathrm{m}^{2}\right)$ & $23.4 \pm 0.6$ & $23.2 \pm 0.6$ \\
Body fat $(\%)$ & $29.9 \pm 1.0$ & $29.5 \pm 0.9$ \\
SBP $(\mathrm{mmHg})$ & $125.7 \pm 3.7$ & $116.9 \pm 3.0^{* *}$ \\
DBP $(\mathrm{mmHg})$ & $75.0 \pm 2.2$ & $71.4 \pm 1.8^{*}$ \\
NOx $(\mu \mathrm{mol} / \mathrm{l})$ & $26.5 \pm 2.3$ & $29.0 \pm 2.3$ \\
PWC75\%HRmax $(\mathrm{w})$ & $24.5 \pm 0.9$ & $28.0 \pm 0.9^{*}$ \\
\hline
\end{tabular}

${ }^{*} \mathrm{p}<0.05$, vs. pre. ${ }^{*} \mathrm{p}<0.01$, vs. pre. Values are mean \pm SEM.

Pre: before 12 weeks of aerobic exercise; Post: after 12 weeks of aerobic exercise; BMI: body mass index; SBP: systolic blood pressure; DBP: diastolic blood pressure; NOx: blood concentrations of nitrogen oxides; PWC75\%HRmax: physical work capacity at $75 \%$ of heart rate maximum.

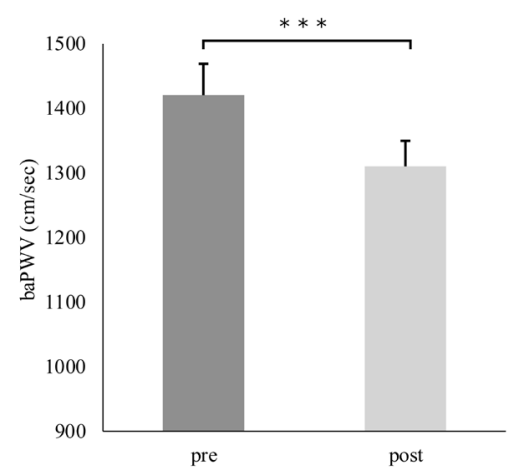

Fig. 1. Time course of baPWV. BaPWV at the post time point significantly decreased as compared to its pre value. $* * * \mathrm{p}<0.001$, significantly lower than pre.

BaPWV: brachial-ankle pulse wave velocity; Pre: before 12 weeks of aerobic exercise; Post: after 12 weeks of aerobic exercise.

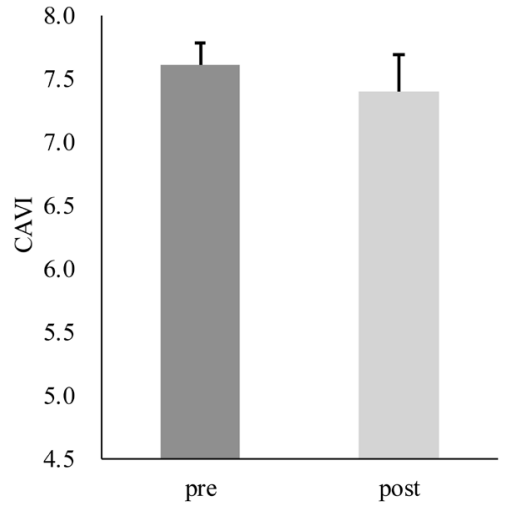

Fig. 2. Time course of CAVI. CAVI was not significantly different between the pre and post time points.

CAVI: cardio-ankle vascular index; Pre: before 12 weeks of aerobic exercise; Post: after 12 weeks of aerobic exercise.

in prehypertensive and hypertensive participants $(159.5 \pm 17.3 \mathrm{~cm} / \mathrm{sec})(\mathrm{p}<0.001)$. BaPWV and CAVI before the exercise intervention was $1420 \mathrm{~cm} / \mathrm{sec}$ and 7.6, respectively.

Evaluation of the relationships between the change in baPWV and blood pressure as well as between the changes in baPWV and NOx revealed a significant correlation only between the change in baPWV and systolic blood pressure $(\mathrm{r}=0.505$, $\mathrm{p}<0.05$ ) (Table 2). More specifically, comparison of these parameters between the pre and post time points revealed that participants with a larger decrease in baPWV showed a larger decrease in systolic blood pressure. No correlation was observed between the change in CAVI and each parameter.

There were significant correlations between the change in baPWV and each of the baseline values for baPWV $(r=0.665$, $\mathrm{p}<0.001)$, SBP $(\mathrm{r}=0.666, \mathrm{p}<0.001)$, and DBP $(\mathrm{r}=0.451, \mathrm{p}<0.05)$ (Table 3). No correlation was observed between the change in CAVI and each of the baseline values for these parameters.

In stepwise multiple regression analysis, R2 was higher when baPWV and SBP were used as explanatory variables $(0.560)$ than when SBP was used as an explanatory variable (0.443) (Table 4).

\section{DISCUSSION}

Among the measures of arterial stiffness, baPWV decreased and CAVI showed no improvement after the 12-week moderateintensity aerobic exercise intervention performed 2-3 times per week. According to our literature search, such a trend in the changes of simultaneously measured baPWV and CAVI has not been previously described. BaPWV and CAVI have been shown to improve after exercise performed once or twice per week ${ }^{15,16)}$. This observation indicates that an adequate frequency of exer- 
Table 2. Relationship of a decrease in arterial stiffness with blood pressure and a decrease in NOx

\begin{tabular}{lccc}
\hline & $\Delta$ SBP & $\Delta$ DBP & $\Delta$ NOx \\
\hline$\Delta$ baPWV & $0.505(0.023)$ & $0.196(0.407)$ & $-0.057(0.812)$ \\
$\Delta$ CAVI & $0.256(0.276)$ & $0.199(0.401)$ & $-0.094(0.693)$ \\
\hline
\end{tabular}

$\mathrm{r}$ (p-value)

BaPWV: brachial-ankle pulse wave velocity; CAVI: cardio-ankle vascular index; SBP: systolic blood pressure; DBP: diastolic blood pressure; NOx: blood concentrations of nitrogen oxides.

Table 3. Relationship between a decrease in arterial stiffness and the baseline value of each parameter

\begin{tabular}{lrrrrr}
\hline & baPWV & CAVI & \multicolumn{1}{c}{ SBP } & \multicolumn{1}{c}{ DBP } & \multicolumn{1}{c}{ NOx } \\
\hline$\Delta$ baPWV & $0.665(0.001)$ & $0.214(0.364)$ & $0.666(0.001)$ & $0.451(0.046)$ & $0.383(0.095)$ \\
$\Delta$ CAVI & $-0.396(0.084)$ & $-0.099(0.679)$ & $-0.540(0.821)$ & $-0.059(0.804)$ & $0.291(0.214)$ \\
\hline
\end{tabular}

$\mathrm{r}$ (p-value)

BaPWV: brachial-ankle pulse wave velocity; CAVI: cardio-ankle vascular index; SBP: systolic blood pressure; DBP: diastolic blood pressure; NOx: blood concentrations of nitrogen oxides.

Table 4. Factors contributing to a decrease in baPWV

\begin{tabular}{lcccc}
\hline & $\mathrm{r}$ & $\mathrm{p}$ & $\beta$ & $\rho$ \\
\hline baPWV & 0.665 & 0.001 & 0.420 & 0.042 \\
CAVI & 0.214 & 0.364 & & \\
SBP & 0.666 & 0.001 & 0.422 & 0.041 \\
DBP & 0.451 & 0.046 & & \\
NOx & 0.838 & 0.095 & & \\
& & & $\mathrm{R}^{2}=0.560$ & \\
\hline
\end{tabular}

BaPWV: brachial-ankle pulse wave velocity; CAVI: cardio-ankle vascular index; SBP: systolic blood pressure; DBP: diastolic blood pressure; NOx: blood concentrations of nitrogen oxides.

cise intervention should be employed in our study. Therefore, the difference in the post-exercise changes in baPWV and CAVI observed between studies may be associated with the differences in participant characteristics, but not in exercise prescription.

One participant characteristic showing inter-participant differences is the baseline value of arterial stiffness parameter. The mean values of baPWV and CAVI in our participants before the exercise intervention were higher (baPWV=1,420 cm/ sec) and lower $(\mathrm{CAVI}=7.6)$ than the age-matched reference values, respectively $\left.{ }^{17,}{ }^{18}\right)$. These observations raised the possibility that exercise could exert remarkable effects only in our participants with high baseline baPWV. In addition, each of our participants with higher baseline baPWV showed a larger post-exercise decrease in baPWV. This observation supports the results from the study on exercise and arterial stiffness first reported by Ikegami ${ }^{19)}$, suggesting a certain level of trainability to be required for improvement of arterial stiffness after exercise.

Another participant characteristic showing the inter-participant difference is the baseline value of SBP. In our study, participants with higher pre-exercise SBP showed higher pre-exercise baPWV as well as a large post-exercise decrease in baPWV; after exercise, participants with a larger decrease in blood pressure showed a larger decrease in baPWV. These observations indicated that SBP should be considered when evaluating the effects of exercise intervention on arterial stiffness. A systematic review performed by Montero et al. has shown that aerobic exercise may exert effects on arterial stiffness in prehypertensive or hypertensive participants only when their SBP values decrease after exercise ${ }^{20}$ ). Among the 20 participants included in our study, 11 with prehypertension or hypertension showed a significantly larger post-exercise decrease in baPWV than did participants with optimal blood pressure. These results supported the previous study reported by Montero et al.

On the other hand, CAVI is a measure of arterial stiffness derived from the theories of stiffness parameter $\beta$ and PMV, which are hardly affected by blood pressure ${ }^{21}$. Shirai et al. reported no association between CAVI and blood pressure $(\mathrm{r}=0.01-0.18)$ as well as a significant association between baPWV and BP $(\mathrm{r}=0.34-0.46)$. It has since been confirmed that CAVI is independent of blood pressure, when compared with the other measures of arterial stiffness ${ }^{5,22,23)}$.

One clue of the mechanism of decreased arterial stiffness, although not completely elucidated, is the existence of nitric oxide $(\mathrm{NO})^{24)}$. A post-exercise increase in the production of $\mathrm{NO}$ as a vasodilator substance has been previously shown using the NOx level as a measure of NO production ${ }^{25,26)}$. However, our study showed no difference in CAVI as an independent measure of arterial stiffness or the NOx level as a measure of NO production between pre- and post-exercise intervention. This result also 
indicated the post-exercise decrease in arterial stiffness observed in our study to be greatly associated with blood pressure.

The results of the present study confirmed that, among the measures of arterial stiffness, including baPWV and CAVI, only baPWV improved after exercise. In addition, participants in whom both blood pressure and CAVI or either alone was higher before exercise showed a larger post-exercise decrease in baPWV. In the future, further confirmation is needed to appropriately use baPWV and CAVI as measures of arterial stiffness on an as-needed basis through identification of similarities and differences between these two measures.

\section{Conflict of interest}

The authors declare that they have no conflict of interest.

\section{REFERENCES}

1) Mitchell GF, Hwang SJ, Vasan RS, et al.: Arterial stiffness and cardiovascular events: the Framingham Heart Study. Circulation, 2010, 121: 505-511. [Medline] [CrossRef]

2) Munakata M, Ito N, Nunokawa T, et al.: Utility of automated brachial ankle pulse wave velocity measurements in hypertensive patients. Am J Hypertens, 2003, 16: 653-657. [Medline] [CrossRef]

3) Vlachopoulos C, Aznaouridis K, Terentes-Printzios D, et al.: Prediction of cardiovascular events and all-cause mortality with brachial-ankle elasticity index: a systematic review and meta-analysis. Hypertension, 2012, 60: 556-562. [Medline] [CrossRef]

4) Hayashi K, Sato M, Niimi H, et al.: [Analysis of the constitutive laws of the vascular wall by finite deformation theory]. Iyodenshi To Seitai Kogaku, 1975, 13: 293-298 (In Japanese). [Medline]

5) Kubozono T, Miyata M, Ueyama K, et al.: Clinical significance and reproducibility of new arterial distensibility index. Circ J, 2007, 71: 89-94. [Medline] [CrossRef]

6) Okura T, Watanabe S, Kurata M, et al.: Relationship between cardio-ankle vascular index (CAVI) and carotid atherosclerosis in patients with essential hypertension. Hypertens Res, 2007, 30: 335-340. [Medline] [CrossRef]

7) Li Y, Bopp M, Botta F, et al.: Lower body vs. upper body resistance training and arterial stiffness in young men. Int J Sports Med, 2015, 36: 960-967. [Medline] [CrossRef]

8) Nishiwaki M, Yonemura H, Kurobe K, et al.: Four weeks of regular static stretching reduces arterial stiffness in middle-aged men. Springerplus, 2015 , 4: 555. [Medline] [CrossRef]

9) Nishiwaki M, Takahara K, Matsumoto N: Arterial stiffness in young adult swimmers. Eur J Appl Physiol, 2017, 117: 131-138. [Medline] [CrossRef]

10) Radtke T, Schmidt-Trucksäss A, Brugger N, et al.: Ultra-endurance sports have no negative impact on indices of arterial stiffness. Eur J Appl Physiol, 2014, 114: 49-57. [Medline] [CrossRef]

11) Satoh N, Shimatsu A, Kato Y, et al.: Evaluation of the cardio-ankle vascular index, a new indicator of arterial stiffness independent of blood pressure, in obesity and metabolic syndrome. Hypertens Res, 2008, 31: 1921-1930. [Medline] [CrossRef]

12) Koshiba H, Maeshima E: Influence of detraining on temporal changes in arterial stiffness in endurance athletes: a prospective study. J Phys Ther Sci, 2015, 27: 3681-3684. [Medline] [CrossRef]

13) Green LC, Wagner DA, Glogowski J, et al.: Analysis of nitrate, nitrite, and [15N]nitrate in biological fluids. Anal Biochem, 1982, 126: 131-138. [Medline] [CrossRef]

14) Miyashita M, Mutoh Y, Yoshioka N, et al.: PWC75\%HRmax: a measure of aerobic work capacity. Sports Med, 1985, 2: 159-164. [Medline] [CrossRef]

15) Nishiwaki M, Fujibayashi M, Nanayama C, et al.: Increasing levels of daily physical activity for arterial stiffness in older women: a community-based pilot study. J Sports Med Phys Fitness, 2018, 58: 1701-1709.

16) Miura H, Nakagawa E, Takahashi Y: Influence of group training frequency on arterial stiffness in elderly women. Eur J Appl Physiol, 2008, 104: 1039-1044. [Medline] [CrossRef]

17) Tomiyama H, Yamashina A, Arai T, et al.: Influences of age and gender on results of noninvasive brachial-ankle pulse wave velocity measurement--a survey of 12517 subjects. Atherosclerosis, 2003, 166: 303-309. [Medline] [CrossRef]

18) Shirai K, Hiruta N, Song M, et al.: Cardio-ankle vascular index (CAVI) as a novel indicator of arterial stiffness: theory, evidence and perspectives. J Atheroscler Thromb, 2011, 18: 924-938. [Medline] [CrossRef]

19) Ikegami H: Effects of physical training on body composition, respire-circulatory functions, blood constituents, and physical abilities. Part 1 : men aged 30 years. Jpn J Phys Fit Sports Med, 1983, 32: 302-309. [CrossRef]

20) Montero D, Roche E, Martinez-Rodriguez A: The impact of aerobic exercise training on arterial stiffness in pre- and hypertensive subjects: a systematic review and meta-analysis. Int J Cardiol, 2014, 173: 361-368. [Medline] [CrossRef]

21) Shirai K, Utino J, Otsuka K, et al.: A novel blood pressure-independent arterial wall stiffness parameter; cardio-ankle vascular index (CAVI). J Atheroscler Thromb, 2006, 13: 101-107. [Medline] [CrossRef]

22) Ibata J, Sasaki H, Kakimoto T, et al.: Cardio-ankle vascular index measures arterial wall stiffness independent of blood pressure. Diabetes Res Clin Pract, 2008, 80: 265-270. [Medline] [CrossRef]

23) Takaki A, Ogawa H, Wakeyama T, et al.: Cardio-ankle vascular index is a new noninvasive parameter of arterial stiffness. Circ J, 2007, 71: 1710-1714. [Medline] [CrossRef]

24) Higashi Y, Yoshizumi M: Exercise and endothelial function: role of endothelium-derived nitric oxide and oxidative stress in healthy subjects and hypertensive patients. Pharmacol Ther, 2004, 102: 87-96. [Medline] [CrossRef]

25) Fujie S, Sato K, Miyamoto-Mikami E, et al.: Reduction of arterial stiffness by exercise training is associated with increasing plasma apelin level in middleaged and older adults. PLoS One, 2014, 9: e93545. [Medline] [CrossRef]

26) Maeda S, Miyauchi T, Kakiyama T, et al.: Effects of exercise training of 8 weeks and detraining on plasma levels of endothelium-derived factors, endothelin-1 and nitric oxide, in healthy young humans. Life Sci, 2001, 69: 1005-1016. [Medline] [CrossRef] 